\title{
SATURATION OF THE PARAMETRIC DECAY INSTABILITY NEAR THE LOWER HYBRID FREQUENCY
}

BY

LIU CHEN AND R, L. BERGER

MASIER

\section{PLASMA PHYSICS LABORATORY}

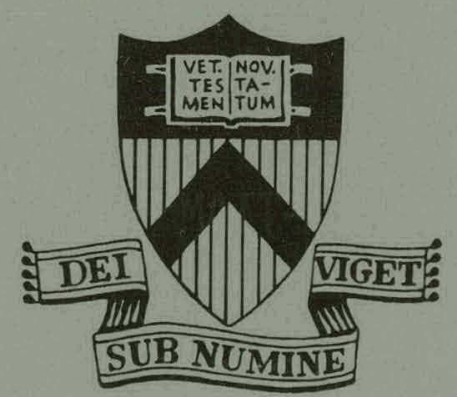

\section{PRINCETON \\ UNIVERS IT Y PRINCETON, NEW JERSEY}

This work was supported by U. S. Energy Research and Development Administracion Contract E (11-1)-3073.

Reproduction, translation, publication, use and disposal, in whole or in part, by or for the United States Government is permitted. 


\section{DISCLAIMER}

This report was prepared as an account of work sponsored by an agency of the United States Government. Neither the United States Government nor any agency Thereof, nor any of their employees, makes any warranty, express or implied, or assumes any legal liability or responsibility for the accuracy, completeness, or usefulness of any information, apparatus, product, or process disclosed, or represents that its use would not infringe privately owned rights. Reference herein to any specific commercial product, process, or service by trade name, trademark, manufacturer, or otherwise does not necessarily constitute or imply its endorsement, recommendation, or favoring by the United States Government or any agency thereof. The views and opinions of authors expressed herein do not necessarily state or reflect those of the United States Government or any agency thereof. 


\section{DISCLAIMER}

Portions of this document may be illegible in electronic image products. Images are produced from the best available original document. 


\section{NOTICE}

This report was prepared as an account of work sponsored by the United States Government. Neither the United States nor the United States Energy Research and Development Administration, nor any of their employees, nor any of their contractors, subcontractors, or their employees, makes any warranty, express or implied, or assumes any legal liability or responsibility for the accuracy, completeness or usefulness of any information, apparatus, product or process disclosed, or represents that its use would not infringe privately owned rights.

Printed in the United States of America.

Available from

National Technical Information Service

U. S. Department of Commerce 5285 Port Royal Road

Springfield, Virginia 22151

Price: Printed Copy $\$$ * ; Microfiche $\$ 1.45$

*Pages

$$
1-50
$$

$51-150$

$151-325$

$326-500$

501-1000
NTIS

Selling Price

$\$ 4.00$

5.45

7.60

10.60

13.60 
Saturation of the Parametric Decay. Instability Near the Lower Hybrid Frequency

Liu Chen and. R. L. Berger

Plasma Physics Laboratory, Princeton University

Princeton, New Jersey 08540

\begin{abstract}
The nonlinear evolution and saturation of parametrically excited lower hybrid waves are studied numerically by solving the corresponding weak turbulence wave kinetic equation. The saturation level and the effective collision frequency $\nu_{f}$ are obtained as functions of pump power.
\end{abstract}

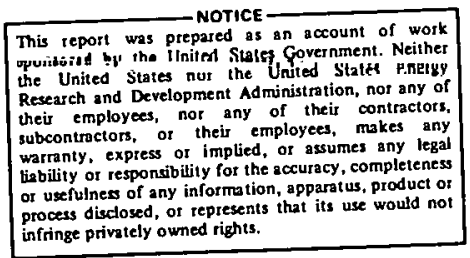


A number of experiments have observed the parametric decay of an externally imposed electric field oscillating at a frequency $\omega_{0}$ close to the lower hybrid frequency $\omega_{1 h} \cdot{ }^{1}$ This decay leads to rapid absorption of energy by plasma waves which ultimately heat the plasma through Landau damping and collisional damping. Since radio frequency power is readily available at frequencies corresponding to the lower hybrid frequency for typical thermonuclear plasmas, this process provides an interesting possibility to heat plasmas to fusion temperatures.

It is necessary to follow the time evolution nf the lower hybrid decay waves to the eventual saturated state in order to predict the rate of energy absorption, total ion and electron heating, and the presence or absence of high energy tails. It is the primary purpose of this paper to study this process.

It has been shown that only twe wave decays are allowed for hot equal temperature plasmas. 2 Thcзc are decay into lower hybrid...waves and either electrostatic ion cyclotron waves or quasimodes. This latter process is nonlinear Landau damping on electrọns (NELD) which dominates the more familiar damping on ions when the ratio of parallel to perpendicular wavenumber is less than the square root of the mass ratio of ions to electrons:. We treat the saturation of the latter instability here even though it has a higher threshold in general than the former decay instability because NELD occupies a larger region of phase space if $\omega_{0}<\sqrt{2} \omega_{1 h}$ and is affected 
less by inhomogeneity than decay into electrostatic ion cyclotron waves.

The pump electric field $\underset{\sim}{\mathrm{E}}(t)$ is taken to be spatially homogeneous, circularly polarized, and perpendicular to the static magnetic field $\underset{\sim O}{B_{O}}=B_{O \sim z}^{e}$, i.e.,

$$
E_{\sim}(t)=E_{O}\left(e_{\sim} \cos \omega_{O} t-{\underset{\sim}{e}}_{y} \sin \omega_{O} t\right),
$$

which corresponds to a fast (whistler) wave pump. Consideration has been restricted to weak pumps so that the maximum parametric growth rate $\gamma_{p}^{m}$ is proportional to the minimum lower hybrid wave damping decrement $\gamma_{e}^{c}$ but is much less than the beat wave damping, i.e.,

$$
1 / 8(\pi / 2 e)^{1 / 2}\left(u_{0}^{2} / c_{s}^{2}\right)\left(\omega_{1 h}^{2} / \omega_{0}^{2}\right)\left(\omega_{k} / \gamma_{e}^{c}\right) \geq 1,
$$

where $u_{0}=E_{0} / B_{0}, c_{s}=\left(T_{e} / m_{i}\right)^{1 / 2}, \omega_{1 h}=\omega_{p i} /\left(1+\omega_{p e}^{2} / \omega_{c e}^{2}\right)^{1 / 2}$, and $\omega_{k}={ }^{(1)} 1 \mathrm{~h}\left(1+\mathrm{m}_{i} k_{\|}^{2} / \mathrm{m}_{\mathrm{e}} \mathrm{k}^{2}+3 \mathrm{k}^{2} \mathrm{v}_{\mathrm{i}}^{2} / \omega_{\mathrm{Ih}}^{2}\right)^{1 / 2}$ is the lower hybrid wave frequency including ion but not electron pressure. Here, $k_{\|}$ is the component of the wavevector parallel to $\underset{\sim}{B}$. The minimum damping decrement is determined by electron ion collisions, i.e., $\gamma_{e}^{e}=v_{e}$, where $v_{e}$ is the collisional damping of lower hybrid waves. The weak turbulence wave kinetic equation governing the evolution of the parametrically excited lower hybrid waves has been derived elsewhere $e^{3,4}$ and is given below. 


$$
(1 / 2)(\partial / \partial t) N(k ; \theta)=\left(\underset{\sim}{\gamma_{k}^{0}}-\underset{\sim}{\gamma_{k}^{D}}-v_{e}+\underset{\sim}{\gamma_{k}^{N L}}\right) N(k, \theta)+s(k, \theta),
$$

where

$$
\begin{aligned}
& N(k, \theta)=2 \pi \varepsilon_{0}\left(1+\omega_{p e}^{2} / \omega_{c e}^{2}\right)|\underset{\sim}{E}(k, \theta)|^{2} /{\underset{\sim}{k}}_{k},
\end{aligned}
$$

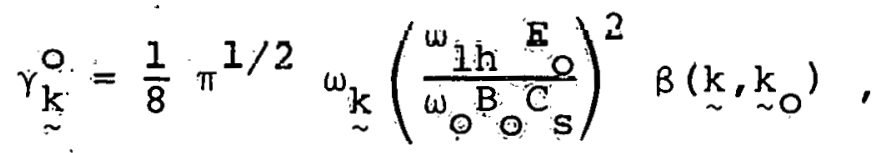

$$
\begin{aligned}
& \underset{\sim}{\gamma_{k}^{N L}}=\frac{1}{4} \pi \underset{\vdots}{1 / 2}\left(\frac{\omega_{1 h}}{B_{0} C_{s}}\right)^{2}\left(1+\frac{\omega_{p e}^{2}}{\omega_{c e}^{2}}\right)^{-1} \cdot \int_{0}^{\infty} d k^{\prime} k^{\prime 2} \\
& \int_{-\pi / 2}^{\pi / 2} d \theta^{\prime} \sin \theta^{\prime} \beta\left(\underset{\sim}{k}, k_{\sim}^{\prime}\right) N\left(k^{\prime}, 0^{\prime}\right),
\end{aligned}
$$

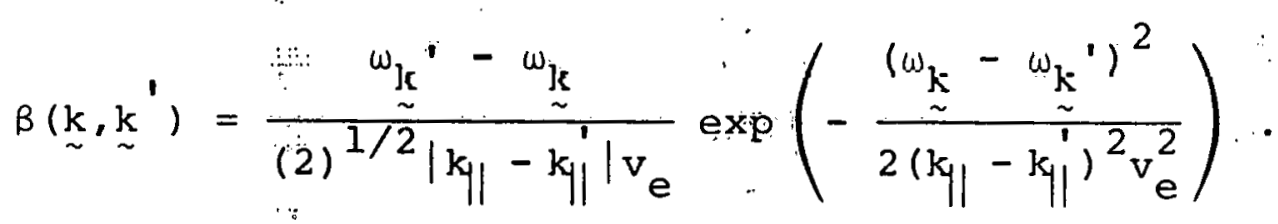

In the above equations, $k_{1}=k \cos \theta, k_{k}$ is the lower hybrid wave frequency, $\gamma_{k}^{0}$ is the linear parametric growth rate for the dipole $\left(k_{\sim}^{k}=0\right)$ pump given in Eq. (1), $\gamma_{k}^{d}$ is the sum of ion and electron Landau damping, and $\gamma_{\sim}^{\mathrm{nl}}$ is the nonlinear damping (growth) due to NELD. We take $\mathrm{\sim}_{0}=0$ and $\omega_{\sim}{ }_{\sim}=\omega_{0} \cdot$ We assumed that $\omega_{c i} \leq\left|\omega_{k}-\omega_{k}\right|<|\underset{\sim}{k}-\underset{\sim}{k}| C_{s}$ in obtaining Eq. (7). 
Equation (3) has been averaged over the azimuthal angle $\psi$ in $\mathrm{k}$ space. The number density of lower hybrid waves or plasmon number $\mathrm{N}_{\mathrm{k}}$ is assumed to be independent of $\psi$ and symmetric about the $\theta=\tilde{\pi} / 2$ plane. The last term in Eq. (3), $\mathrm{s}_{\underset{\sim}{k}}$, is the spontaneous emission which we set equal to a small constant. In some cases the value of $s_{k}$ has been varied by an order of magnitude without altering the results essentially. It is easy to show from Eq. (7) that the wave energy density $\varepsilon_{\underset{\sim}{\mathbf{k}}}=|| \omega_{\underset{\sim}{\mathbf{k}}} \mid \mathrm{N}_{\underset{\sim}{\mathrm{k}}}$ flows in time from high to low frequency modes. We also see from Eq. (7) that $B\left(\underset{\sim}{\mathrm{k}}, \mathrm{\sim}_{\sim}^{\prime}\right)$ is antisymmetric with respect to the interchange of $\underset{\sim}{\mathrm{k}}$ and $\mathrm{k}_{\sim}^{\prime}$. As a consequence, the total number of plasmons and the total wave energy are conserved by NELD. Moreover, Kruer and $\mathrm{Valeo}^{5}$ have shown for an equation equivalent to Eq. (3) that a steady state solution of Eq. (3) is accessible and unique and for which the supply of energy by the pump is balanced by the loss of energy to particles.

Equation (3) is solved numerically by dividing wavenumber spare into a discrete number of nodes with a number of modes represented in the characteristic widths of the matrix element $\Delta \mathrm{k} / \mathrm{k} \sim \mathrm{n} /\left(\mathrm{kC}_{\mathrm{s}} / \omega_{\mathrm{LH}}\right)$ and $\Delta \mathrm{n} \sim \mathrm{kC}_{\mathrm{s}} / \omega_{\mathrm{LH}}$ where $n=\left(\mathrm{m}_{i} / \mathrm{m}_{\mathrm{e}}\right)^{1 / 2} \cos \theta$. The integral in Eq. (3) is replaced by a sum such that the volume element in $\underset{\sim}{k}$ space is a constant. Wavenumber space is divided. into a rectangular grid such that $\eta<1$ and $\mathrm{k}_{\text {Illin }}<\mathrm{k}<\mathrm{k}_{\max }$. The maximum value of $\mathrm{k}, \mathrm{k}_{\max }$, is chosen such that there are no growing modes with this wavenumber. The 
minimum value of $k$ is determined by $k_{\max }$ and the $k$ division. $A$ total of 300 modes with 20 divisions of $n$ and 15 divisions of $\mathrm{k}$ were used to integrate Eq. (3). The time step was chosen to be small compared to the growth time of the instability. The evolution of the normalized field energy density $\mathrm{w}_{\mathrm{k}}=\left|\mathrm{E}_{\mathrm{k}}\right|^{2} / \mathrm{E}_{\mathrm{o}}^{2}$ was followed in time for values of pump intensity corresponding to $1.3,1.5,1.7,2.0$, and 3.0 times threshold intensity. In all cases we set $\mathrm{T}_{\mathrm{e}}=\mathrm{T}_{i}, \omega_{\mathrm{pe}}=\omega_{c e}$, and $\omega_{0}=\omega_{p i}=\sqrt{2} \omega_{L H} \because$ Linearly unstable modes exponentiate in time and transfer energy to lower frequency modes. The total decay wave energy :saturates when the number of plasmons created by the pump equals the number of plasmons dissipated by Landau and collisional damping. Since the frequency of the lower hybrid mode and the Landau damping àre increasing functions of $\mathrm{k}$, the nonlinear saturated state will be characterized by a concentration of energy at small $\mathrm{k}$ values. Because the energy density at small $\mathrm{k}$. is dissipated primarily by collisions, the main body electrons are heated.

Figure 1 shows the time evolution of the total decay wave energy $w=\sum_{k}\left|\mathrm{E}_{\mathrm{k}}\right|^{2 \% / 2 \mathrm{E}_{\mathrm{o}}^{2}}$ for a pump intensity 1.3 times threshold. There is an initial overshoot followed by an oscillatory approach to a steady state value. This is a general feature of the time evolution of the spectrum. In Fig. 2 , the two dimensional saturated energy density $w_{k}$ is shown for a pump intensity 1.3 times threshold. The energy is concentrated in the linearly 
unstable region where the decay wave energy is barely large enough to exceed the threshold for growth of small $k$ and small $n$ modes, i.e., lower frequency modes. As the pump intensity is raised, additional modes with lower frequency are destabilized by the linearly unstable modes. At pump intensity 2.0 times threshold, shown in Fig. 3, the decay wave energy density is enhanced even near the lowest frequency mode in the system. The energy density for a pump 3.0 times threshold is shown in.Fig. 4. The intensity of the lowest frequency mode in the system has been enhanced several orders of magnitude to a level comparable, with the linearly unstable modes. This mode cannot decay and thus condensation of energy appears likely in the lowest frequency mode for a pump only a few times threshold. Another feature of higher pump intensities is the concentration of plasmons in small wavenumbers $k$ for all values of $n$ because the frequency is a decreasing function of $k$ as well as $\eta$.

Once the energy in the lowest frequency mode is comparable to the energy in higher frequency modes, this saturation mechanism breaks down. We can estimate the pump intensity for which this occurs as follows. Summing over all modes and using the antisymmetric properties of the matrix element $\beta\left(\underset{\sim}{\left.\mathbf{k}, \mathbf{\sim}_{\sim}^{\prime}\right)}\right.$ we find from Eq. (3)

$$
\frac{\partial}{\partial \dot{\tau}} \mathrm{N}_{\mathrm{I} I}=\sum_{k} 2 \gamma_{\mathrm{o}}(k, \Phi) \mathrm{N}(\mathrm{k}, \Phi) \cdots \nu_{\mathrm{e}} \mathrm{N}_{\mathrm{T}}
$$


where $\mathrm{N}_{\mathrm{T}}$ is the total number of plasmons. We define an average growth rate $\bar{\gamma}_{0}$ such that $\bar{\gamma}_{0} N_{1} \equiv \sum_{k} 2 \gamma_{0}(k, \Phi) N(k, \Phi)$ where $N_{1}$ is the total number of linearly unstable modes. An upper limit on the ratio of $\mathrm{N}_{\mathrm{T}}$ to $\mathrm{N}_{1}$ can be estimated by the ratio of the total phase space, $A_{T}$, occupied by modes with enhanced intensity to the phase space $A^{\vdots}$, occupied by $\mathrm{N}_{1}$. Defining a steady state by setting $\partial N_{T} / \partial \dot{t}=0$ and using Eq. (8), we find that $\overline{\bar{\gamma}}_{\mathrm{O}} / \nu_{\mathrm{e}}<\mathrm{N}_{\mathrm{T}} / \mathrm{N}_{1}<\dot{\mathrm{A}}_{\mathrm{T}} / \mathrm{A}_{1}$. The ratio $\mathrm{A}_{\mathrm{T}} / \mathrm{A}_{1}$ is $2-3$ and thus only very weak pumps saturate according to weak turbulence theory. In Fig. 5, the total field energy at saturation $W$ normalized to the pump amplitude squared is plotted versus $E_{0}^{2} / E_{t h}^{2}$ where $E_{t h}$ is the threshold pump amplitude. The results indicate an approximately linear proportionality, i.e., $W=\sum_{k}\left|E_{k}\right|^{2} / 2 E_{0}^{2} \simeq 0.2 E_{o}^{2} / E_{t h}^{2}$. The effective collision frequency is dẽfined by

$$
\begin{aligned}
& 1 / \overline{2} \nu_{f} \varepsilon_{0}\left\langle\left|E_{\sim}(t)\right|^{2}\right\rangle=\nu_{f} \varepsilon_{o} E_{o}^{2}
\end{aligned}
$$

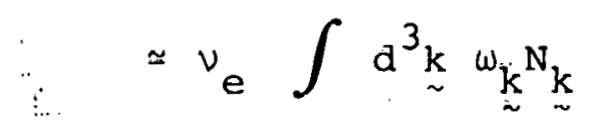

$$
\begin{aligned}
& =\left(\because+\frac{\omega_{p e}^{2}}{\omega_{c e}^{2}}\right) v_{e}\left(\varepsilon_{o} E_{o}^{2}\right) \sum_{k}\left|E_{\sim}^{k}\right|^{2} / 2 E_{o}^{2} .
\end{aligned}
$$

Hence, we find that the effective collision frequency is proportional to $\mathrm{E}_{0}^{2}$, i.e., 


$$
\nu_{f}=0.8 \nu_{e} E_{o}^{2} / E_{t h}^{2}
$$

The linear dependence of $\nu_{f}$ on the pump power is characteristic. of weak turbulence saturation mechanisms. Following arguments similar to Galeev and Sagdeev, ${ }^{6}$ we can estimate the effective collision frequency by setting $\nu_{f}$ equal to the maximum paxametric growth rate $\gamma_{\mathrm{k}}^{\circ}$. That is, we assume that the instability will stop growing when the damping decrement on the fastest growing mode is raised to the level such that the pump is at threshold again. This estimate agrees with the, numerical scaling law given in Eq. (9). Within the limits of weak turbulence theory, the predicted heating rate is not enhanced much above classical collisional absorption because $\left(E_{0} / E_{t h}\right)^{2}<3$. Moreover, at saturation, the decay wave energy is concentrated in long wavelength waves which are damped by electron-ion collisions. This leads to main body electron heating.

\section{ACKNOWLEDGMENTS}

The authors wish to thank Drs. F. W. Perkins and C. Oberman for helpful discussions.

This work was supported by U. S. Energy Research and Development Administration Contrart F: $(11-1)=3073$. 


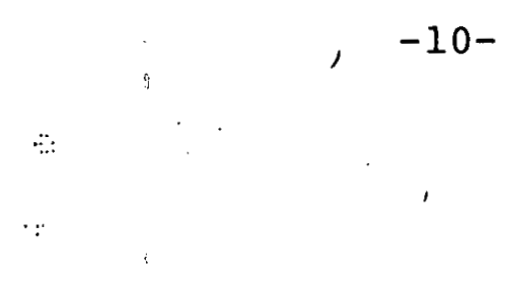

REFERENCES

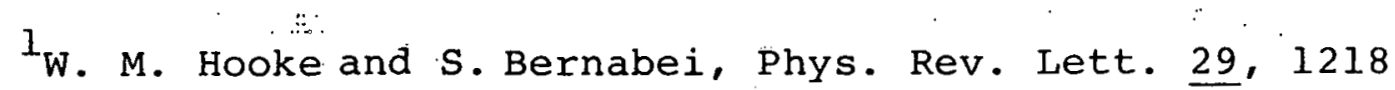
(1972); T. K. Chü, S. Bernabei, and R. W. Motley, Phys. Rev. Lett. 31, 211. (1973); R. P. H. Chang and M. Porkolab, Phys. Rev. Lett. $32,1227(1974)$.

${ }^{2}$ R. L. Berger and F. W. Perkins, Princeton University Report No. PPL-MATT-1975 (to be published in Phys. Fluids).

${ }^{3}$ A. Hasegawa and I. Chen, (to be published in Phys. Fluids oct. 1975).

${ }^{4}$ A. Rogister, Phys. Rev. Lett. 34,80 (1975).

${ }^{5}$ W. L. Kruer and E. J. Valeo, Phys. Fluids 16, 675 (1973).

${ }^{6}$ A. A. Galeev and R. Z. Sagdeev, Nuclear Fusion 13, 603 (1973). 


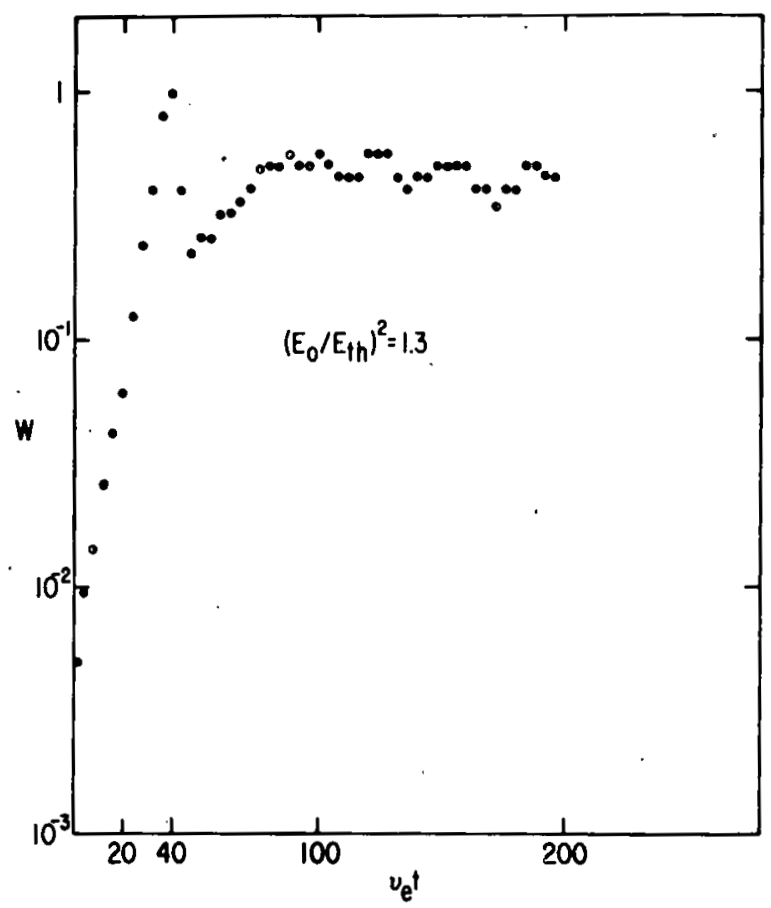

752220

Fig. 1. Plot of the total field energy in decay modes normalized to the pump amplitude squared versus time in units of inverse electron-ion collision frequency for a pump power I.3 times threshold power.

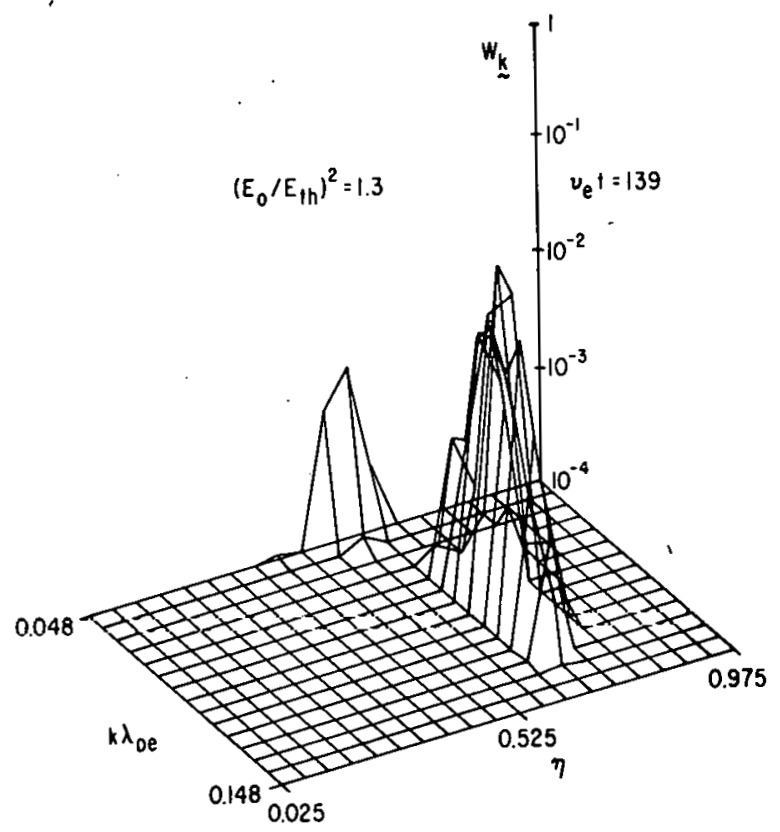

752222

Fig. 2. The normalized field energy density $W(k, \theta)$ at time $v_{e i t}=139$ after saturation (see Fig. 1) for a pump power 1.3 times threshold plotted as a function of $n=\left(m_{i} / m_{e}\right)^{1 / 2} k_{\|} / \mathrm{k}$ and $\mathrm{k} \lambda_{\mathrm{De}} \cdot$ 


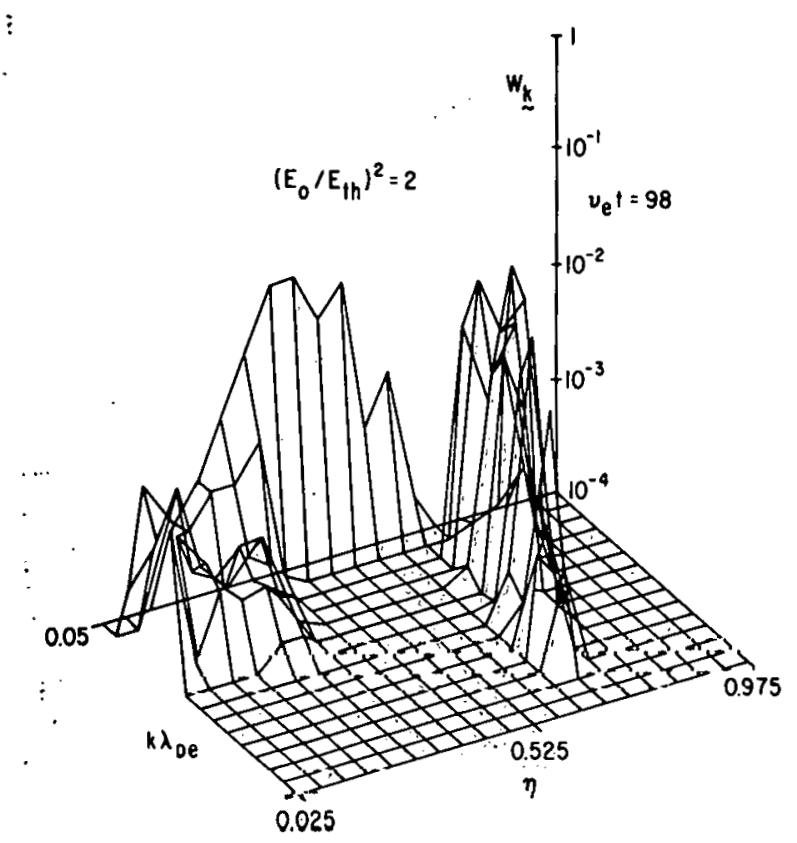

752221

F19. 3. l'he saturated field energy density $W(k, \theta)$ at time $\nu_{\text {eit }}=98$ for a pump power 2.0 times threshold plotted as a function of $n=\left(m_{i} / m_{e}\right)^{1 / 2} k_{\|} / k$ and $k \lambda$ De

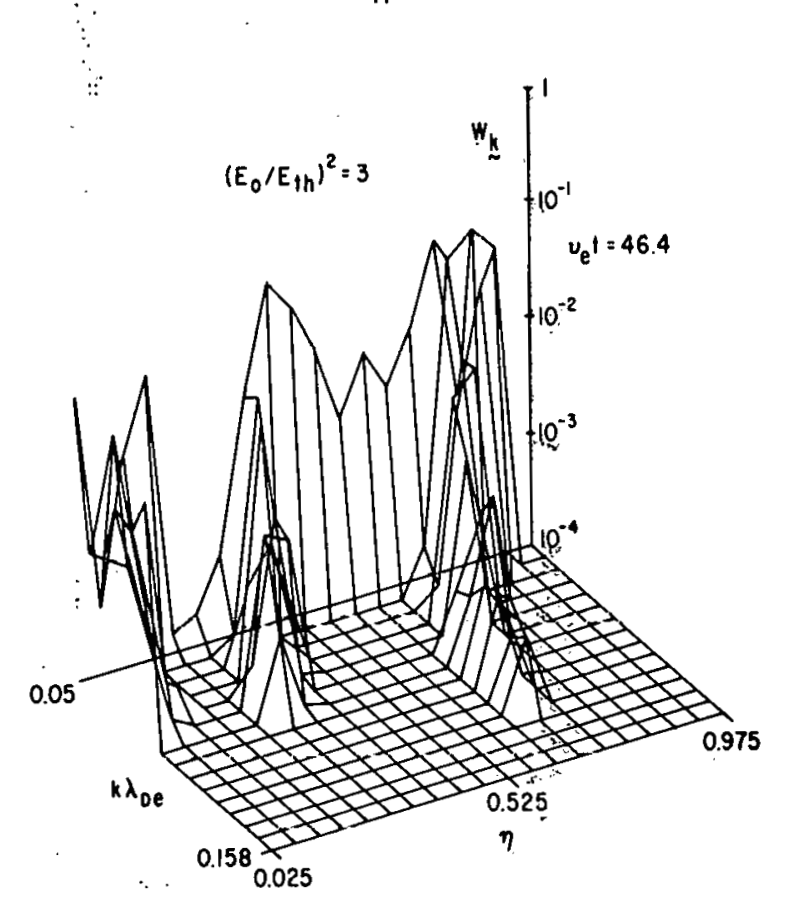

752223

Fig. 4. The saturated field energy density $w_{k}$ at time $\hat{Y}_{e i}=46.4$ for a pump power 3 times threshold ${ }^{k}$ plotted as a Eunction of $n=\left(m_{i} / m_{e}\right)^{1 / 2} k_{\|} / \vec{k}$ and $k \lambda$ De. 


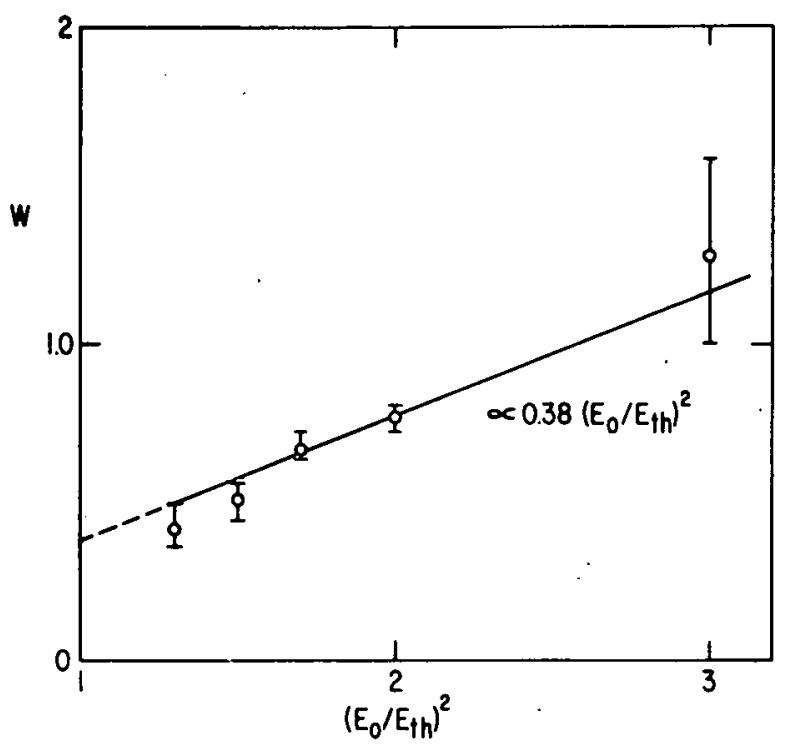

752224

Fig. 5. The total field energy $W=\sum_{\mathbf{k}} W_{\mathbf{k}}$ normalized by the pump amplitude squared plotted vers̃us the pump power normalized to threshold power. (Note: No cases were run for $\left(E_{o} / E_{t h}\right)^{2}<1.3$ and the initial energy lies far below the intersection of the dotted line with the ordinate.) 